\title{
Erratum: Digital Certificate Management for Document Workflows in E-Government Services
}

Florin Pop, Ciprian Dobre, Decebal Popescu, Vlad Ciobanu, and Valentin Cristea

Computer Science Department, Faculty of Automatic Control and Computers

University POLITEHNICA of Bucharest, Romania

\{florin.pop, ciprian.dobre, decebal.popescu, vlad.ciobanu, valentin.cristea\}@e-caesar.ro

DOI: 10.1007/978-3-642-14799-9_37

Section 2 "Related Work" of the paper "Digital Certificate Management for Document Workflows in E-Government Services" starting on page 364 of this book is not comprehensive and offers a general survey only. At the time of writing, the authors were unaware of the official support for EJBCA offered by PrimeKey Solutions AB. There are both free and professional PKI support for EJBCA and several government institutions in Europe and private companies around the world have bought professional support services provided by PrimeKey.

The original online version for this chapter can be found at http://dx.doi.org/10.1007/978-3-642-14799-9 31 